\title{
XVII.
}

(Aus dem Institut für Krebsforschung in Berlin. Direktor: Wirkl. Geh. Rat Prof. Dr. von Leyden, Exz.)

\section{Die Katalasewirkung in normaler und in carcinomatöser Leber.}

\author{
Voll
}

Prof. Dr. Ferdinand Blumenthal

und Dr. rer. nat. B. Brahn, Assistent am Institut für lirebsforsehung.

Von jeher bildete es eine der wichtigsten Aufgaben der Krebsforschung, festzustellen, ob die den Krebsgeschwülsten innewohnenden malignen Eigenschaften bedingt sind durch das Vorhandensein fremder Lebewesen, welche von aussen in den Körper eindrangen (parasitäre Theorie), oder ob die Gewebe selbst zum Teil ihre Widerstandsfähigkeit gegen den natürlichen Wachstumstrieb der Epithelzellen, welcher ihnen innewohnen soll, verloren haben, wodurch dann ein schrankenloses Wachstum der Epithelzellen auf Kosten der übrigen Gewebszellen möglich sei, eine Auffassung, welche in den Untersuchungen von v. Leyden und Bergell ihre experimentelle Stütze gefunden hat. Diese letztere Auffassung vermag sicherlich das Wachstum der Krebsgeschwülste, auch die Metastasenbildung, bis zu einem gewissen Grade zu erklären. Zur Erklärung anderer und wichtigerer maligner Eigenschaften der Krebszelle selbst müssen Veränderungen in ihr gegenüber der normalen Epithelzelle hinzukommen, wodurch sie befähigt wird, ihr Zerstörungswerk im Organismus auszuführen.

Die Feststellung dieser Tatsache, worin der Neuerwerb der Krebszelle an biologischen Funktionen bestehen soll. ist wiederholt Gegenstand der Untersuchung gewesen. Wir erinnern an die von Petry festgestellte Tatsache, dass die Autolyse der Krebsgeschwülste vermehrt sei, ferner daran, 
dass Asz̧ites von Krebskranken ein autolytisches Ferment enthält (Umber), welches sonst im Aszites fehlt; ferner an die gleichzeitig von Neuberg einerseits und von Blumenthal und Wolff andererseits gefundene Tatsache, dass Krebsgeschwülste häufig die Fähigkeit haben, Gewebe anderer Organe abzubauen ${ }^{1}$ ). Dazu kommt die von Blumenthal festgestellte Schwerverdaulichkeit des Krebsgewebes durch Pepsin und die Leichtverdaulichkeit durch Pankreatin, während die normalen Gewebe ein umgekehrtes Verhalten zeigen. Hier muss es sich teilweise um einen Neuerwerb von Eigenschaften handeln, welche mit zur Erklärung der der Krebszelle im Gegensatz zu anderen Zellen des Körpers innewohnenden Eigenschaften berangezogen werden können. In gleichem Sinne bewegen sich Mitteilungen, wonach die Krebsgeschwülste im Gegensatz zu den Geweben, in denen sie sich entwickelt haben, einen Verlust zeigen an katalytischer und oxydativer Wirkung, worüber wir bisher kurz in der Medizinischen Klinik 1909. No. 1 berichtet haben.

Vergleichende Untersuchungen über die Intensität der Oxydation im Organismus und dem Katalasengehalt des Blutes hat $\mathrm{Jolles}^{2}$ ) angestellt und auch dabei quantitative Methoden zur Bestimmung der Katalasenwirkung angegeben. Uns fiel der Unterschied in der katalytischen Wirkung normaler and krebsiger Leber auf. Wir untersuchten die hier obwaltenden quantitativen Unterschiede und kamen zu folgenden Resultaten:

1. Setzt man frische normale Leber und frische einer Leber entnommene Krebsknoten der Autolyse aus und stellt durch etwa einen Monat deren katalytische Wirkungen quantitativ fest, so fällt sofort die gewaltig grössere katalytische Wirkung der normalen Leber gegenüber derjenigen der Krebsknoten auf. Die normale Leber zersetzte die Wasserstoffsuperoxydlösung unter stürmischem Aufbrausen, während die Krebsknoten nur mässige Gasentwicklung hervorriefen;

2. die zweite auffallende Erscheinung war, dass die Katalasezahlen der normalen Leber sich in der etwa vierwöchigen Versuchszeit nur ganz unwesentlich änderten, während die viel niedrigeren Zablen des Carcinoms in dieser Zeit noch bedeutend geringer wurden;

3. das wichtigste Ergebnis unserer Untersuchung schien uns aber, dass auch die gesunden Teile einer nur wenige Krebsknoten ent-

1) Da die Arbeit bereits im Juli 1909 fertiggestellt war, so konnte die neueste Literatur nicht berücksichtigt werden.

2) Ueber die quantitative Bestimmung der Katalasen im Blut. Vortrag auf der Naturforscher-Versammlung 1904, Breslau. 
haltenden Leber eine viel geringere Katalasenwirkung liatten als normale Leber, dass hier also eine Schwächung des katalytischen Ferments in den gesunden Teilen einer Krebsleber durch den kranken Teil vorlag;

4. wurden Mischungen normaler und Krebsleber auf ihre katalytischen Wirkungen untersucht; man fand nur Mischverhältnisse, also keine Wirkungen von Antifermenten;

5. wurden auch Sarkome auf ihre katalytische Fermententwicklung hin untersucht und hier ein noch geringeres katalytisches Vermögen als beim Carcinom gefunden.

Es folgen nun die Versuchsanordnnngen im einzelnen und die erhaltenen Zahlen.

\section{Normale Leber.}

Je $50 \mathrm{~g}$ frische, möglichst fein zerkleinerte Leber wurden mit $500 \mathrm{~g}$ Chloroformwasser bei $37^{\circ}$ im Brutschrank der Autolyse ausgesetzt. Nach bestimmten Fristen wurden der Autolyseflüssigkeit Proben von je $5 \mathrm{ccm}$ mit der Pipette entnommen und mit einem Ueberschuss $(10 \mathrm{ccm})$ von Wasserstoffsuperoxyd zwei Stunden stehen gelassen. Dann wurden die Nischungen mit $1 / 10$ proz. Kaliumpermanganatlösung so lange titriert, bis eben eine dauernde Rotfärbung eintrat. $10 \mathrm{~cm}$ der gebrauchten $\mathrm{H}_{2} \mathrm{O}_{2}$ Lösung entfärbten $206,5 \mathrm{ccm} 1 / 10$ proz. $\mathrm{KMnO}_{4}$. Je grösser also die bei der Titration bis zum Eintritt der Rotfärbung noch nötige Menge $\mathrm{KMnO}_{4}{ }^{-}$ Lösung war, desto geringer die Menge, bzw. die Wirkung der in der Leber vorbandenen Katalase.

Tabelle I. Normale Leber.

\begin{tabular}{|c|c|c|c|}
\hline \multirow{2}{*}{$\begin{array}{l}\text { Dauer der } \\
\text { Autolyse }\end{array}$} & Leber I & Leber 2 & Leber 3 \\
\hline & \multicolumn{3}{|c|}{$\operatorname{ccm} 1 / 10$ proz. $\mathrm{KMnO}_{4}$} \\
\hline 1 Stunde & 16,3 & 14,0 & 8,1 \\
\hline $1 \mathrm{Tag}$ & 8,6 & 12,1 & 8,0 \\
\hline 3 Tage & 8,4 & 9,4 & 5,2 \\
\hline 5 & 8,5 & 9,3 & 7,6 \\
\hline & 8,0 & 10,0 & 11,9 \\
\hline 9 & 10,1 & 9,4 & 10,4 \\
\hline 11 & 9,4 & 11,6 & 11,4 \\
\hline 16 & 12,3 & 12,3 & 16,0 \\
\hline 21 & 13,1 & 12,9 & 14,9 \\
\hline 26 & 16,2 & 14,1 & 23,2 \\
\hline $31 \%$ & 15,9 & 15,2 & 24,7 \\
\hline
\end{tabular}




\section{Versuche mit Lebercarcinomen.}

Statt der Leber wurde je $\tilde{0} \mathrm{~g}$ frisch herausgeschältes, möglichst von normalen Teilen freies, sehr fein zerkleinertes Lebercarcinom verwandt. Sonst alles wie bei den Versuchen mit normaler Leber.

Tabelle II. Carcinom.

\begin{tabular}{|c|c|c|c|c|c|c|c|c|}
\hline \multirow{2}{*}{$\begin{array}{l}\text { Dauer der } \\
\text { Autolyse }\end{array}$} & Carc. 1 & Care. 2 & Care. 3 & Care. 4 & Carc. 5 & Carc. 6 & Care. 7 & Carc. 8 \\
\hline & \multicolumn{8}{|c|}{$\mathrm{cem} \mathrm{1/10} \mathrm{proz.} \mathrm{KMnO}_{4}$} \\
\hline 1 Stunde & 159,4 & 145,3 & 146,0 & 154,1 & 149,5 & 146,2 & 173,0 & 162,1 \\
\hline 1 Tag & 141,3 & 144,2 & 142,0 & 153,0 & 150,1 & 139,1 & 154,7 & 148,9 \\
\hline 3 Tage & 142,1 & 148,1 & 141.7 & 153,9 & 154,3 & 138,4 & 161,2 & 157,1 \\
\hline$\overline{0} \quad$. & 150,2 & 139,2 & 149,1 & 160.1 & 156,4 & 141,0 & 159.8 & 154,3 \\
\hline $7 \quad$. & 146,3 & 142,5 & 150,4 & 158,8 & 155,9 & 150,2 & 162,0 & 162,1 \\
\hline 9 & 153,8 & 150,1 & - & - & - & - & - & - \\
\hline 10 & - & - & 156,1 & 164,3 & 159,4 & 146,3 & 163,0 & 161,8 \\
\hline 13 & 159,1 & 164,3 & - & - & - & - & - & - \\
\hline 15 & 164,2 & 165,7 & 175,2 & 171,2 & 164,1 & 152,1 & 171,5 & 165,7 \\
\hline 20 & 170,5 & 181,2 & 178,3 & 182,3 & 166,3 & 155,7 & 170,2 & 165,8 \\
\hline 25 & 179,4 & 185,4 & 195,1 & 181,9 & 174,3 & 162,3 & 179,5 & 182.5 \\
\hline 30 & 181,2 & 190,0 & 192,0 & 184,3 & 190,0 & 170,4 & 178,4 & 185,5 \\
\hline $45 \quad$. & 185,8 & 194,2 & 198,0 & 189,7 & 198,3 & 182,0 & 185,3 & 186,1 \\
\hline
\end{tabular}

3. Gesunder Teil einer mit Krebsknoten durchsetzten Leber. Versuchsanordnung wie vorher.

Tabelle III. Gesunder Toil von Krebslebern.

\begin{tabular}{|c|c|c|c|c|c|c|}
\hline Dauer der & 1 & 2 & 3 & 4 & 5 & 6 \\
\hline Autoryse & \multicolumn{6}{|c|}{ cem 1/10 proz. $\mathrm{KMnO}_{4}$} \\
\hline 1 Stunde & 117,7 & 92,1 & 131,0 & 103,4 & 95,1 & 84,4 \\
\hline $1 \mathrm{Tag}$ & 116,1 & 93,4 & 132,0 & 115,4 & 94,3 & 79,1 \\
\hline 3 T'age & 116,0 & 100,1 & 134,1 & 124,1 & 94,2 & 78,2 \\
\hline $5 \quad .$. & 118,4 & 98,4 & 138,4 & 129,2 & 96,3 & 80,9 \\
\hline 7 & 125,8 & 98,4 & 137,9 & 129,1 & 102,1 & 79,8 \\
\hline 10 & 125,1 & 99,8 & 137,8 & 129,2 & 101,7 & 91,4 \\
\hline 15 & 131,2 & 110.0 & 142,1 & 130,0 & 112,1 & 91,0 \\
\hline 20 & 132,9 & 112,3 & 151,2 & 140,4 & 113,3 & 102,4 \\
\hline 25 & 140,0 & 111,0 & 157,1 & 137,1 & 117,0 & 107,3 \\
\hline & 143,1 & 117,0 & 157,9 & 140,6 & 121,4 & 108,1 \\
\hline 45 & 144,3 & 124,3 & 162,1 & 141,2 & 124,9 & 117,4 \\
\hline
\end{tabular}


4. Mischungen von je $25 \mathrm{~g}$ Carcinom und $25 \mathrm{~g}$ gesunder Leber. Versuchsanordnung wie vorher.

Tabelle IV. Mischung vongleichen Teilen normaler Leber und Krebsleber.

\begin{tabular}{c|c|c}
\hline \multirow{2}{*}{$\begin{array}{c}\text { Dauer der } \\
\text { Autolyse }\end{array}$} & Versuch 1 & Versuch 2 \\
\cline { 2 - 3 } & ccm 1/10 proz. $\mathrm{KMnO}_{4}$ \\
\hline & & \\
1 Stunde & 94,1 & 104,0 \\
1 Tag & 90,0 & 98,0 \\
5 Tage & 92,3 & 109,7 \\
10 & 95,3 & 107,1 \\
20 & 96,2 & 118,9 \\
30 & 107,8 & 130,1
\end{tabular}

5. Je $30 \mathrm{~g}$ reines Sarkom mit $300 \mathrm{~g}$ Chloroformwasser. Sonst wie vorher.

Tabelle V. Sarkome.

\begin{tabular}{|c|c|c|}
\hline \multirow{2}{*}{$\begin{array}{l}\text { Dauer der } \\
\text { Autolyse }\end{array}$} & Sarkom 1 & Sarkom 2 \\
\hline & \multicolumn{2}{|c|}{$\operatorname{cen} 1 / 10$ proz. $\mathrm{KMnO}_{4}$} \\
\hline 1 Stunde & 198,1 & 201,0 \\
\hline $1 \mathrm{Tag}$ & 198,0 & 199,4 \\
\hline 5 Tage & 199,2 & 200,3 \\
\hline 10 & 199,5 & 201,2 \\
\hline $20 "$ & 203,1 & 201,9 \\
\hline 30 & 203,4 & 204,0 \\
\hline
\end{tabular}

